\title{
Adipose tissue-derived mesenchymal stem cells differentiated into hepatocyte-like cells in vivo and in vitro
}

\author{
LIBO YIN, YUHUA ZHU, JIANGANG YANG, YIJIANG NI, \\ ZHAO ZHOU, YU CHEN and LIXING WEN \\ Department of Traumatic Surgery, Changzhou No. 2 People's Hospital, Nanjing Medical University, \\ Changzhou, Jiangsu 213000, P.R. China
}

Received December 27, 2013; Accepted July 22, 2014

DOI: $10.3892 / \mathrm{mmr} .2014 .2935$

\begin{abstract}
Cell-based therapy is a potential alternative to liver transplantation. The goal of the present study was to examine the in vivo and in vitro hepatic differentiation potential of adipose tissue-derived mesenchymal stem cells (AT-MSCs) and to explore its therapeutic use. AT-MSCs were isolated and cultured with hepatic differentiation medium. Bioactivity assays were used to study the properties of AT-MSCs. The morphology of differentiated AT-MSCs in serum-free hepatic differentiation medium changed into polygonal epithelial cells, while the morphology of AT-MSCs in a similar medium containing $2 \%$ fetal bovine serum remained unchanged. The differentiated cells cultured without serum showed hepatocyte-like cell morphology and hepatocyte-specific markers, including albumin (ALB) and $\alpha$-fetoprotein. The bioactivity assays revealed that hepatocyte-like cells could take up low-density lipoprotein (LDL) and store glycogen. Furthermore, trichostatin A (TSA) enhanced ALB production and LDL uptake by the hepatocyte-like cells, analogous to the functions of human liver cells. ALB was detected in the livers of the $\mathrm{CCl}_{4}$-injured mice one month post-transplantation. This suggested that transplantation of the human AT-MSCs could relieve the impairment of acute $\mathrm{CCl}_{4}$-injured livers in nude mice. This therefore implied that adipose tissue was a source of multipotent stem cells which had the potential to differentiate into mature, transplantable hepatocyte-like cells in vivo and in vitro. In addition, the present study determined that TSA was essential to promoting differentiation of human MSC towards functional hepatocyte-like cells. The relief of liver injury following treatment with AT-MSCs suggested their potential as a novel therapeutic method for liver disorders or injury.
\end{abstract}

Correspondence to: Dr Lixing Wen, Department of Traumatic Surgery, Changzhou No. 2 People's Hospital, Nanjing Medical University, 29 Xinglong Lane, Changzhou, Jiangsu 213000, P.R. China

E-mail: xiaominwang39@163.com

Key words: differentiation, adipose tissue, mesenchymal stem cells, liver injury, cell

\section{Introduction}

Certain liver diseases lead to hepatocyte damage that can progress to liver failure, for which a transplant may be the patients' only treatment option. However, transplant organs are a highly limited resource, and transplant rejection remains to be an unresolved problem. The replacement of hepatocytes by stem cells or stem cell-stimulated endogenous and exogenous regeneration are the major goals of liver-directed cell therapy (1-4). Mature proliferating hepatocytes have the potential to be used as hepatic cell replacement (5). However, hepatocyte progenitors are still required under certain circumstances, such as the impaired ability of differentiated hepatocytes to further divide (6). Studies have suggested that reservoirs of stem cells may reside in organ tissues in order to promote self-repair and regeneration (7). However, adult stem cells have been suggested to be more plastic than once believed (8). Adult stem cells can be isolated from human lipoaspirates and differentiate toward osteogenic, adipogenic, neurogenic, myogenic and chondrogenic lineages (9). While stem cells derived from different tissues have shown promise for therapeutic applications, Lagasse et al (10) demonstrated that transplanted purified hematopoietic stem cells could give rise to hepatocytes and restore liver function in fumarylacetoacetate hydrolase-deficient mice. In humans, female recipients of male bone marrow (BM) were found to have hepatocytes that contained the Y chromosome (11), implying that hepatocytes could be derived from BM cells (12). Several studies have indicated that transplanted BM cells adopt the phenotype of hepatocytes and restore liver function by cell fusion rather than differentiation $(13,14)$. Kern et al (3) and Wagner et al (15) compared mesenchymal stem cells (MSCs) derived from human adipose and bone marrow with respect to morphology, the success rate of isolating MSCs, colony frequency, expansion potential, multiple differentiation capacity and immune phenotype. They showed that adipose tissue-derived mesenchymal stem cells (AT-MSCs) had similar characteristics to those of BM mesenchymal stem cells (BMSCs). Adipose tissue contains stem cells similar to BMSCs; these cells could be isolated from cosmetic liposuctions and grown easily under standard tissue culture conditions (3). The multi-lineage differentiation capacity of AT-MSCs cells has been confirmed $(3,9,15)$. 
The aim of the present study was to investigate whether AT-MSCs had the potential to differentiate into functional hepatocytes in vitro and in vivo, in order to provide a novel stem cell therapy for the treatment of liver diseases.

\section{Materials and methods}

All procedures were performed according to manufacturer's instruction unless noted otherwise.

Isolation and culture of MSCs from adipose tissue. Human adipose tissue was collected following liposuction surgery performed at Zhongshan Hospital affiliated to Xiamen University (Xiamen, China). Written informed consent and approval were obtained from the patients or their family, and the Human Research Ethical Committee of Zhongshan Hospital Xiamen University (Xiamen, China). The lipoaspirates $(10-20 \mathrm{ml})$ were washed twice with an equal volume of phosphate-buffered saline (PBS; Digestive Diseases Institute of Xiamen University, Xiamen, China) and digested with $0.075 \%$ collagenase I (Sigma, St. Louis, MO, USA). Red Blood Cell Lysis Buffer (Beyotime, Haimen, China) was used to eliminate erythrocytes; cells were passed through a $40-\mu \mathrm{m}$ mesh filter, and suspended in low-glucose Dulbecco's modified Eagle's medium (DMEM-LG; Gibco-BLR, Carlsbad, CA, USA) supplemented with $10 \%$ fetal bovine serum (FBS) and $100 \mathrm{U} / \mathrm{ml}$ penicillin/streptomycin (Gibco-BLR). The resuspended stromal vascular fraction (SVF) cells were plated at a density of $5 \times 10^{5} / \mathrm{cm}^{2}$ in a $100-\mathrm{mm}$ culture dish (Corning; Corning, NY, USA). The fibroblastoid adherent cells were designated AT-MSCs. Cells were harvested at $90 \%$ confluence using Trypsin-EDTA (Gibco-BLR), and designated as passage $0(\mathrm{P} 0)$. Cells at P3-P5 were used for the subsequent experiments unless otherwise stated.

Growth kinetics of AT-MSCs. To determine the growth kinetics of cultured AT-MSCs, 60-mm culture dishes were seeded with $1 \times 10^{5}$ cultured AT-MSCs (P3-P5). At several time-points (between days 2 and 12) following seeding, cells from duplicate dishes were harvested and counted. AT-MSC numbers were plotted against the number of days in culture, and the exponential growth phase of the cells was determined.

Measurement of AT-MSC proliferation. For the cell proliferation assay, $2 \times 10^{3}$ viable AT-MSC were seeded in each triplicate well in a 96-well plate. AT-MSCs proliferation was measured using a cell counting kit 8 (CCK-8; Beyotime). The plates were placed in a humidified incubator until the cells adhered to the plate; then $10 \mu \mathrm{l}$ of the CCK- 8 solution was added to each well and plates were incubated for another $2 \mathrm{~h}$ at $37^{\circ} \mathrm{C}$ prior to reading the absorbance at $450 \mathrm{~nm}$ using a microplate reader (550; Bio-rad Laboratories, Inc., Shanghai, China). The assay was repeated every day at the same time for one week.

Cell cycle analysis. AT-MSCs in the exponential growth phase were detached with $0.25 \%$ trypsin-EDTA (Gibco-BRL), and the resultant pelleted AT-MSCs $\left(10^{6}\right)$ were gently suspended in $1 \mathrm{ml} 70 \%$ ethanol (Xiamen Chemical Company, Xiamen, China) and kept for at least $6 \mathrm{~h}$ at $-20^{\circ} \mathrm{C}$. Cells were washed with PBS twice, incubated for $1 \mathrm{~h}$ at room temperature (RT) in the dark with $100 \mu 12.5 \mathrm{mg} / \mathrm{ml}$ propidium iodide (PI) (Sigma-Aldrich, Shanghai, China) and $1 \mathrm{mg} / \mathrm{ml}$ RNase (Beyotime) in PBS. The cells were analyzed with a FACSscan flow cytometer (Beckman Coulter, Brea, CA, USA) with a $488 \mathrm{~nm}$ wavelength.

Transmission electron microscopy. Cells at 80-90\% confluence were harvested with $0.25 \%$ trypsin-EDTA and washed twice with cold PBS. Fixing solution (2.5\% glutaraldehyde; Xiamen Xinlongda Chemicals Company, Xiamen, China) was added and the pellet was incubated for $4 \mathrm{~h}$ at $4^{\circ} \mathrm{C}$. The cells were washed after $2 \mathrm{~h}$ (or overnight) at $4^{\circ} \mathrm{C}$ with $0.1 \mathrm{M}$ PBS three times. The fixed samples, which could be stored stably for several months, were sent to the electron microscopy department in the School of Life Sciences at the Xiamen University for further processing and analysis using the JEM-2100HC transmission electron microscope (JEOL, Tokyo, Japan).

Flow cytometry. AT-MSCs $\left(10^{6}\right)$ were trypsinized and incubated with fluorescein isothiocyanate (FITC)-conjugated CD34, CD45, CD90, phycoerythrin (PE) -conjugated human leukocyte antigen (HLA)-DR, CD11b, CD29, CD105 (mouse, monoclonal, 1:200; eBioscience, San Diego, CA, USA) or PE-conjugated CD3, CD73, CD117, (mouse, monoclonal, 1:200; BD Biosciences, San Jose, CA, USA) antibodies for 30 minutes at RT, followed by three washes. Staining with unconjugated anti-programmed death ligand (PDL) 1 (rabbit, polyclonal, 1:100; BD Biosciences), FITC-conjugated goat anti-rabbit immunoglobulin $\mathrm{G}(\mathrm{IgG})$ antibody (Ab) (goat, polyclonal, 1:200; BD Biosciences) was used as a secondary antibody. The fluorescent labeled cells were analyzed on a FACSscan flow cytometer (Becton-Dickinson, Franklin Lakes, NJ, USA) using CELLQuest Pro software (Becton-Dickinson).

Immunofluorescence assay (IFA). Cultured cells were fixed with $4 \%$ paraformaldehyde (Sigma) in PBS for 5 minutes at RT and permeabilized with $0.3 \%$ Triton X-100 (Sigma) for 20 minutes. Cells were then incubated with blocking solution consisting of PBS and 5\% FBS at RT for 30 minutes. For immunofluorescence staining, primary antibodies against albumin (ALB), (rat, polyclonal, 1:50; Santa Cruz Biotechnology, Inc., Santa Cruz, CA, USA), $\alpha$-fetoprotein (AFP) (mouse, mono, 1:50; Wuhan Boster Biological Technology, Ltd., Wuhan, China) and pan cytokeratin (panCK; mouse, mono, 1:50; Zhongshan Goldenbridge Biotechnology, Beijing, China) were used. Following incubation with the primary antibody, cells were incubated with FITC-labeled anti-rat, Dylight-conjugated anti-mouse or FITC-labeled anti-mouse (rabbit, polyclonal, 1:200; Jackson ImmunoResearch, West Grove, PA, USA) secondary antibodies for $1 \mathrm{~h}$ at $37^{\circ} \mathrm{C}$ and stained with DAPI (Sigma) to identify the cell nuclei. The cells were photographed with a structured illumination fluorescence microscope (DM IL LED; Leica Microsystems, Tokyo, Japan).

Hepatic differentiation protocols. To induce hepatogenic differentiation, AT-MSCs were plated after three passages on 5-mm culture dishes coated with collagen type I (Sigma) in expansion medium (DMEM-LG supplemented with $10 \%$ FBS). Following reaching confluence, cells were washed twice with PBS and cultured in basic hepatic 


\section{Heptogenic differentiation protocal}

A

$\stackrel{100 \% \text { confluency }}{\longrightarrow}$ Differentiation

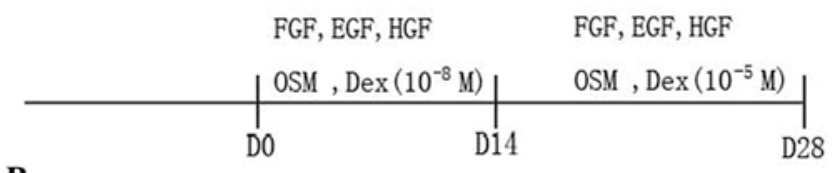

B

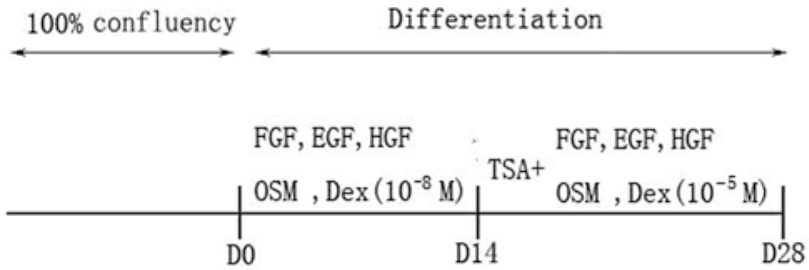

Figure 1. Schematic presentation of the hepatic differentiation protocols. All cells were pre-cultured in expansion medium until $100 \%$ confluence was achieved. (A) AT-MSCs were exposed to hepatic inducing agents at day 0 ; on day 14 , dexamethasone concentration was changed from low $\left(10^{-8} \mathrm{M}\right)$ to high $\left(10^{-5} \mathrm{M}\right)$. (B) Protocol A with the addition of TSA on day 14 . The media changed every two days; hepatic differentiation was then assessed at different time-points. F/E/HGF, fibroblast/epidermal/hepatocyte growth factor; D, day; OSM, oncostatin M; Dex, dexamethasone; TSA, trichostatin A.

differentiation medium supplemented with $1 \mathrm{X}$ insulin-transferrin-selenium (ITS; Sigma), $10^{-8} \mathrm{M}$ dexamethasone (Sigma), $20 \mathrm{ng} / \mathrm{ml}$ epidermal growth factor (PeproTech EC, London, UK), $20 \mathrm{ng} / \mathrm{ml}$ fibroblast growth factor (FGF; PeproTech EC), $40 \mathrm{ng} / \mathrm{ml}$ oncostatin M (OSM; Sigma) and $40 \mathrm{ng} / \mathrm{ml}$ hepatocyte growth factor (HGF) (PeproTech EC). After 2 weeks, the medium was replaced with hepatic differentiation medium with an increased concentration of dexamethasone at $10^{-5} \mathrm{M}$ (Fig. 1A) and/or $1 \mu \mathrm{M}$ trichostatin A (TSA; Sigma) (Fig. 1B). The differentiation medium was used with or without serum. $2 \mathrm{ml}$ of differentiation medium was added to each 12 -well culture dish and changed twice a week. Hepatic differentiation was identified by cell morphology, immunohistochemistry, reverse transcription polymerase chain reaction (RT-PCR) analysis and biochemical functions at different time-points. All assays were performed with undifferentiated AT-MSCs as negative controls and human primary hepatocytes or HepG2 cells (Life and Science College, Xiamen University, Xiamen, China) as positive controls.

In vitro adipogenic differentiation. The adipogenic differentiation assay was performed on AT-MSCs obtained between P3-P5 using the Human Mesenchymal Stem Adipogenic Differentiation Medium kit (Cyagen Biosciences, Guangzhou, China). Induction medium was used until the cells reached $100 \%$ confluence or post-confluence. Three days after confluence, the medium was changed to maintenance medium; $24 \mathrm{~h}$ later it was changed back to induction medium. Following completing three cycles of induction and maintenance, the cells were incubated for a further seven days in the adipogenic maintenance medium. The non-induced control cells were fed only with adipogenic maintenance medium. Adipogenic differentiation was confirmed by the formation of neutral lipid-vacuoles stainable with Oil Red O (Sigma). Non-induced cells were used as a control.
In vitro osteogenic differentiation. Cells were treated with osteogenic medium (Osteogenic Differentiation kit; Cyagen Biosciences) for two weeks and the induction medium was changed every three days. Osteogenesis was assessed by von Kossa staining (Cyagen Biosciences). Non-induced cells were used as a control.

Total RNA was isolated from hepatic differentiated AT-MSCs (Trizol; Invitrogen, Carlsbad, CA, USA); $2 \mu \mathrm{g}$ total RNA was used for reverse transcription (RevertAid First Strand cDNA Synthesis Kit; Fermentas, Burlington, Ontario, Canada). The cDNA was amplified using LaTaq (Takara BIO, Otsu, Shiga, Japan). Primers, synthetized by Sangon Biotech (Shanghai, China), were used to correspond with human gene sequences (Genbank database, www.ncbi.nlm.nih. gov/genbank):ALBsense,5'-CCCCAAGTGTCAACTCCAA-3' and antisense, 5'-AAAGCAGGTCTCCTTATCGT-3'; AFP sense, 5'-GGCTGACATTATTATCGGACAC-3' and antisense, 5'-GTTCCTCTGTTATTTGTGGC-3'; and $\beta$-actin sense, 5'-TGAAGGTCGGAGTCAACGGATTTGGT-3' and antisense, 5'-CAT GTGGGCCATGAGGTCCACCAC-3' were used as an internal control for PCR. Amplification reactions were performed using an Eppendorf thermocycler (Mastercycler Nexus Gradient, Eppendorf, Germany) at $94^{\circ} \mathrm{C}$ for 30 seconds, $55^{\circ} \mathrm{C}$ for 30 seconds and $72^{\circ} \mathrm{C}$ for 60 seconds for 35 cycles. The PCR products were then separated by electrophoresis on $1.5 \%$ agarose gels (Sigma). The PCR sequencing product was confirmed by automatic sequencing (ABI 3730XL; Applied Biosystems, Foster City, CA, USA).

Periodic acid-Schiff (PAS) staining (Beyotime) was used for the detection of glycogen storage in hepatogenic differentiated cells. Cells were fixed with $10 \%$ formaldehyde (Beyotime) oxidized in $1 \%$ periodic acid (Beyotime) for 10 minutes and rinsed twice with water. Subsequently, cells were treated with Schiff's reagent (Beyotime) for 10 minutes and then rinsed with water.

Uptake of low-density lipoprotein. The uptake of lipoprotein was detected with the Dil-Ac-LDL staining kit (Biomedical Technologies, Stoughton, MA, USA).

Animal model and cell transplantation. Animal experiments were conducted with permission from the Ethical Committee for Animal Experimentation of Xiamen University (Xiamen China) and according to P.R. China legislation. Immune-deficient BALB/c nude mice were obtained from the National Rodent Laboratory Animal Resources, (Shanghai, China); the animals were kept in animal house of Xiamen University according to internationally accepted principles. Animals were housed under standard laboratory conditions of light (12-h light/dark cycle) at $25 \pm 2^{\circ} \mathrm{C}$, with a humidity of $55 \pm 5 \%$, rats were fed a standard mice pellet diet and tap water ad libitum. All the mice were male. Mice were administered a single abdominal injection of olive oil containing $10 \%$ carbon tetrachloride $\left(\mathrm{CCl}_{4}\right.$; Xiamen Chemical Company) at a dose of $100 \mu \mathrm{l} / 20 \mathrm{~g}$ body weight (bw). Cells for transplantation or control solutions were injected into the tail vein: Group A control mice were injected with $100 \mu 110 \% \mathrm{CCl}_{4} / 20 \mathrm{~g}$ bw only (n=9), group B received $100 \mu \mathrm{l}$ PBS $(\mathrm{n}=9)$ and group $\mathrm{C}$ received $100 \mu \mathrm{l}$ PBS containing AT-MSCs $\left(5 \times 10^{5}\right.$ cells $)(n=9)$. Following this procedure, three mice from each group were sacrificed on days 
A

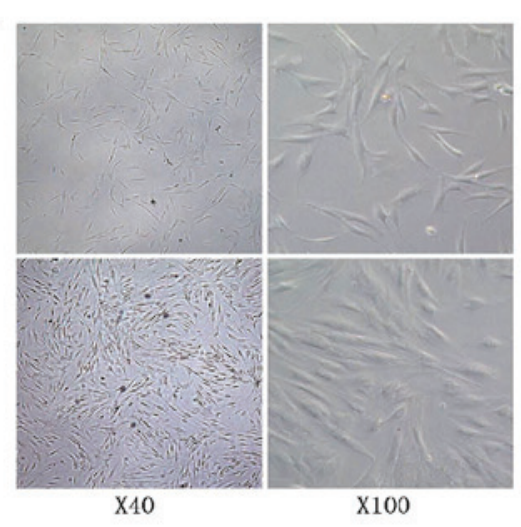

D

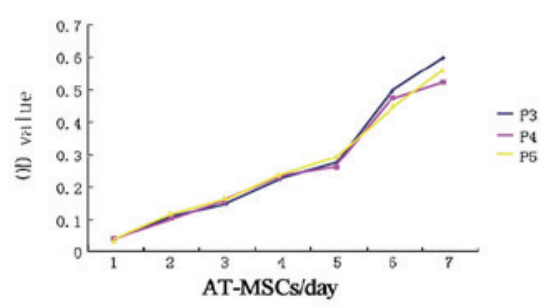

B

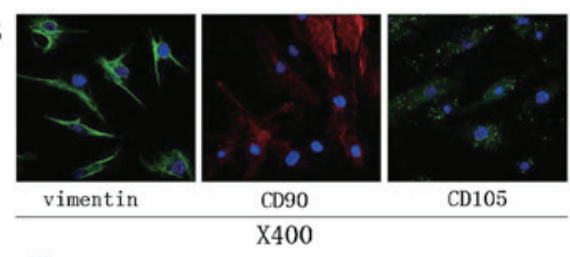

C

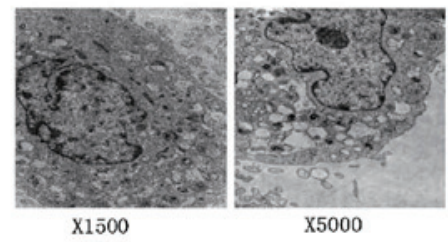

E

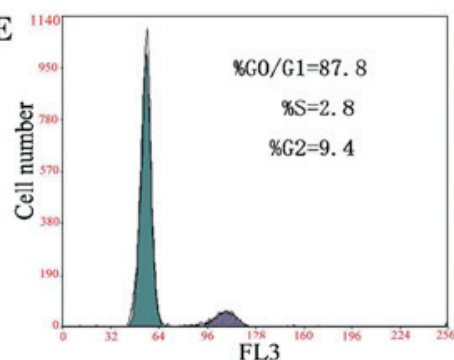

Figure 2. AT-MSC morphology. (A) AT-MSCs showed a fibroblast-like morphology, forming a CFU-F upon confluence. (B) Cells were stained for 1) vimentin and CD90 (FITC, green), 2) CD105 (Dylight, red), and 3) nuclei stained with DAPI. (C) Ultramicrostructure of AT-MSCs: Organelles had a naïve profile. (D) CCK-8 detection of growth kinetics; AT-MSCs of P3-5 had similar characteristics. (E) Cell cycle analysis showed that most cells were in dormant phase. CFU-F, colony forming unit fibroblast; FITC, fluorescein isothiocyanate; AT-MSCs, adipose tissue-derived mesenchymal stem cells; P, passage; OD, optical density.

one, three and seven. Blood samples were collected and whole livers were removed, fixed and prepared for further analysis. The serum concentrations of ammonia, alanine aminotransferase (ALT), aspartate aminotransferase (AST) and direct bilirubin (DBIL) were detected by histological analysis of liver tissue at days one, three and seven. To evaluate engraftment of AT-MSCs in the liver, two additional groups of mice were used: Group I mice were administered $100 \mu 1 / 20 \mathrm{~g}$ bw of $10 \% \mathrm{CCl}_{4}$, followed by AT-MSCs $\left(5 \times 10^{5}\right.$ cells) after $24 \mathrm{~h}$ by injection into the tail vein $(n=3)$; group II mice were injected with AT-MSCs $\left(5 \times 10^{5}\right.$ cells) only $(\mathrm{n}=3)$. One month later, these mice were sacrificed, and the livers were removed and fixed for further study. Histological analysis of liver tissues was conducted by serial tissue sectioning and staining with hematoxylin and eosin (H\&E; Beyotime) or immunohistochemical examination for human-specific ALB expression, as described above.

Two-way mixed lymphocyte reaction (MLR) assay. The two-way MLR assay was used in order to detect whether lymphocyte aggregation was inhibited by AT-MSCs. The MLR was performed in 96-well microtiter plates using RPMI 1640 (Gibco-BRL) medium supplemented with 10\% FBS. Purified T cells derived from two different donors were plated at $2 \times 10^{5}$ cells per donor per well. Peripheral blood mononuclear cells (PBMCs) from two different donors were used as the 'responder cells' for the MLR. PBMCs were prepared by centrifugation of leukapheresed peripheral blood cells (Innovadyne, Santa Rosa, CA, USA). The derived lymphocytes were tested using flow cytometry with antibodies for CD3 and CD19 (BD Biosciences). T cells were mixed in complete culture medium at $2 \times 10^{5}$ cells per donor per well in 96-well microtiter plates. Adipose tissue-derived cells were added to the MLR at 1, 250, 2,500, 5,000 or 10,000 cells per well. Stimulator cells were irradiated with 5,000 rads of $\gamma$-radiation with a cesium irradiator (XH BRI-1000; Baxter, Shanghai, China) prior to being added to the culture wells at the various concentrations. Control cultures consisted of T cells from two donors plated in medium alone (no stimulator cells). Triplicate cultures were set up for each condition. Cultures were pulsed with bromodeoxyuridine (BRDU; Millipore) on day 5 for $10 \mathrm{~h}$. The optical density (OD) of the plates was then read with a spectrophotometer microplate reader (Biorad) set at a wavelength of $450 \mathrm{~nm}$. All the above steps were performed at RT. The percentage of suppression was calculated using the following formula: Percentage suppression $=(1-[$ (Test cell + MLR OD value) $\div$ MLR OD value]) $\times 100 \%$.

Statistical analysis. Results are expressed as mean \pm standard deviation. Statistical analyses were performed using least significant difference (LSD) t-test after one-way analysis of variance (ANOVA) or Student's t-test. $\mathrm{P}<0.05$ was considered to indicate a statistically significant difference between values.

\section{Results}

Characterization of AT-MSCs.

Morphology and ultramicrostructure of AT-MSCs. AT-MSCs were cultivated from the mononuclear cell fraction of adipose tissue samples obtained from healthy donors. 

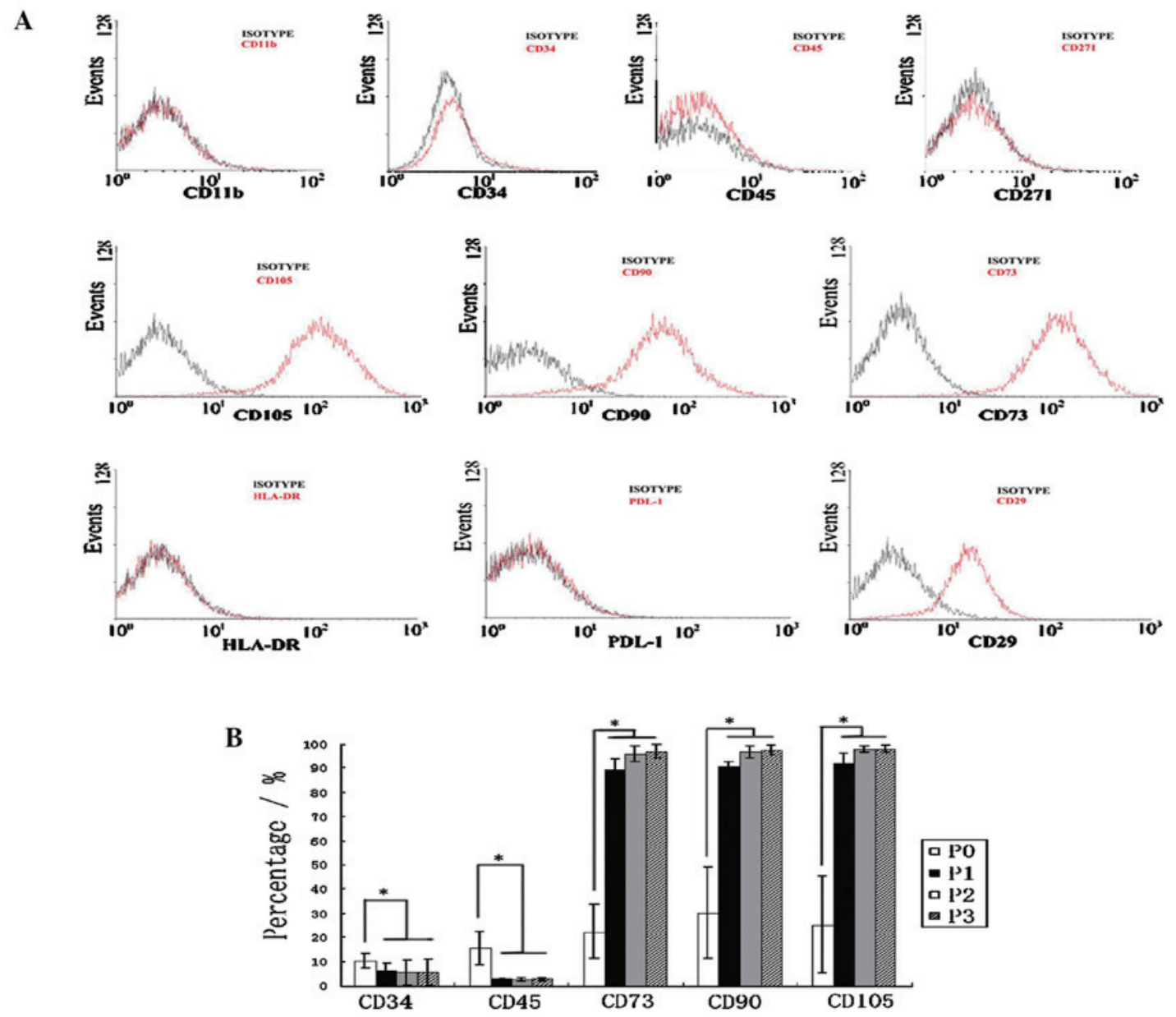

Figure 3. AT-MSCs express a unique set of CD markers. (A) Flow cytometric analysis of the expression of multiple CD antigens (red lines, representative histograms; black lines, respective isotype controls). (B) Changes in expression of CD markers at different passages. Values represent mean \pm standard error of the mean $(n=6)$. Data were analyzed by LSD-t-test following a one way analysis of varience. The expression of all markers shown at P0 differed significantly from that shown at P1/P2/P3 ( $\mathrm{P}<0.05$, LSD-t-test); with no difference in CD marker levels at P1, P2, P3 (P>0.05, LSD-t test). AT-MSCs, adipose tissue-derived mesenchymal stem cells; P, passage; HLA-DR, human leukocyte antigen-DR; PDL-1, programmed death-ligand 1; LSD, least significant difference.

Cells were selected based on plastic adherence to ensure the removal of any contaminating hematopoietic cells, AT-MSCs expanded easily in vitro and exhibited a fibroblast-like morphology (Fig. 2A). The expression of mesenchymal stem cell markers, detected by immunofluorescence, was high in cultured AT-MSCs. The majority of cultured AT-MSCs expressed vimentin, and $>90 \%$ highly expressed CD 90 and CD105 (Fig. 2B). Following subsequent passages, differentiated cells displayed homogeneous morphologies and high rates of proliferation. Examination of AT-MSCs by electron microscopy displayed the presence of numerous surface microvilli. However, it also revealed a limited presence of organelles, including Golgi bodies, rough endoplasmic reticula, mitochondria; by contrast, the differentiated cells showed significant presence of organelles, including plate-like bodies (Fig. 2C).

Cell cycle and growth patterns. AT-MSCs at P3-P5, showed a dynamic growth pattern, with duplication time of $3.00 \pm 0.28$ days. In direct proliferation experiments, AT-MSCs of different passages (P3-P5) showed similar biological characteristics (Fig. 2D) and a stage of rapid cell proliferation approximately five days following cell culture (Fig. 2E). The patterns of proliferation as well as the cell cycle profiles demonstrated that these AT-MSCs displayed classical stem cell features.

Phenotypic characterization of AT-MSC populations. Cell surface markers of AT-MSCs at P3-P5 were analyzed by flow cytometry. The average expression of the following markers from cells of all donors $(n=6)$ were: CD11b (2 $\pm 0.4 \%)$, HLA-DR (3.4 $\pm 0.8 \%)$, PDL-1 $(1.4 \pm 0.4 \%)$, CD29 (96 $\pm 1.3 \%)$, CD34 (5.5 $\pm 5.2 \%)$, CD45 $(2.6 \pm 0.7 \%)$,

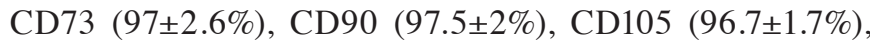
CD271 (2.3 $\pm 1.2 \%)$ (Fig. 3A). These results confirmed that the AT-MSCs expressed characteristic stem cell-associated surface markers CD29, CD73, CD90, CD105, while lacking expression of CD34, CD45, HLA-DR and PDL-1 (Fig. 3A). The hematopoietic lineage markers CD34, CD45 and other markers CD90, CD105 and CD73 were observed by flow cytometry in subsequent cultures of AT-MSCs. These markers were considered the minimum criteria for MSCs. Expression of the MSC markers was found to differ among passages. Of note, AT-MSCs of passage 0, AT-MSCs that were separated from human adipose tissue without cell culture, expressed higher CD34 and CD45 and lower CD73, CD90 and CD105. With increasing time of AT-MSCs in culture, hematopoietic 


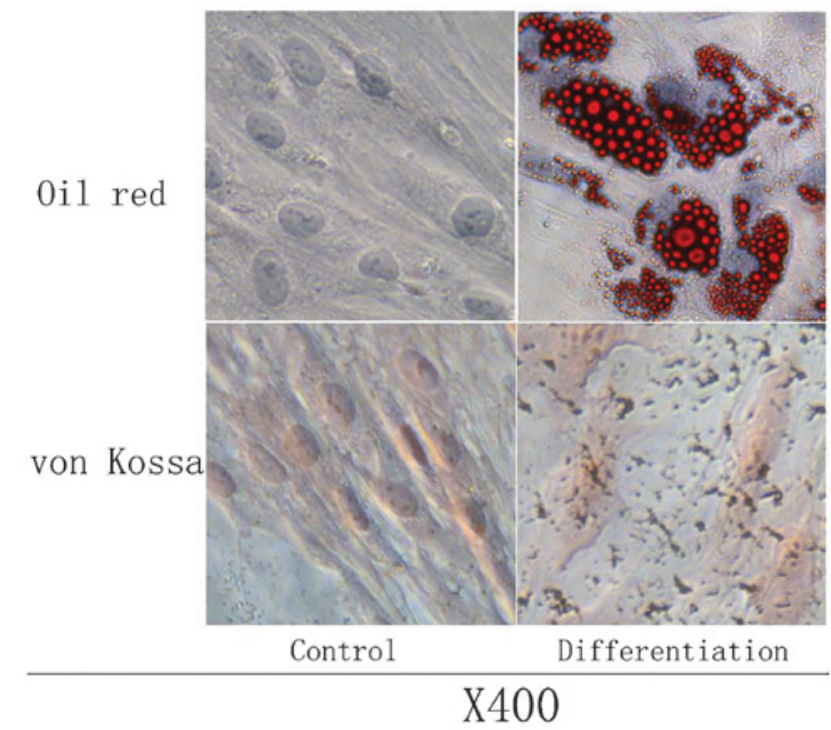

Figure 4. Multi-differentiation capacity of AT-MSCs. Evaluation of adipogenic differentiation of AT-MSCs: Oil Red O staining for the presence of intracytoplasmic lipid-rich droplets. Evaluation of osteogenic differentiation: Extracellular calcium mineralization following osteogenic differentiation stained black; nuclei stained with neural red (von Kossa). No staining observed in untreated AT-MSCs for either condition. AT-MSCs, adipose tissue-derived mesenchymal stem cells.

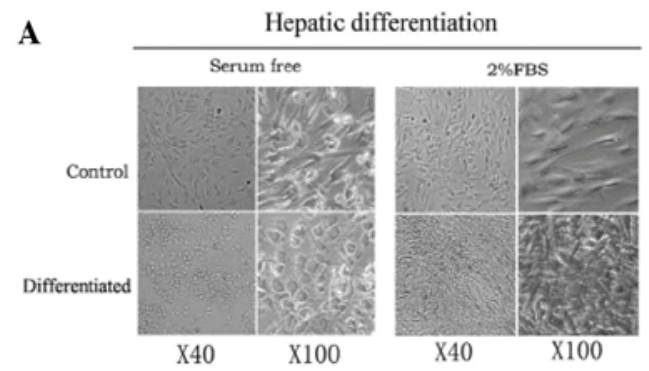

B

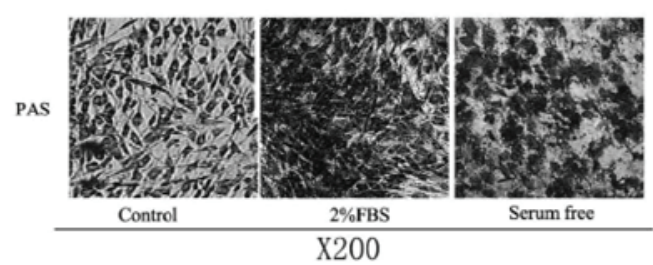

Figure 5. Hepatic differentiation of human AT-MSCs cultured in differentiation medium with or without serum for 14 days. (A) Glycogen storage ability was detected by PAS staining. (B) Morphological changes (polygonal and round shapes) in cells grown in medium without serum; no change in cells grown in medium plus $2 \%$ FBS. AT-MSCs, adipose tissue-derived mesenchymal stem cells; PAS, periodic acid-Schiff stain; FBS, fetal bovine serum.

lineage markers (CD34, CD45) were decreased, while expression of CD73, CD90 and CD105 intensified (Fig. 3B). Therefore, $\mathrm{SVF}$ in $\mathrm{P} 0$ expressed significantly different marker profiles from that of AT-MSCs at P1-P3 $(\mathrm{P}<0.05$, one-way ANOVA and $\mathrm{P}<0.05$, LSD-t-test).

Multi-differentiation capacity of AT-MSCs. The osteogenic and adipogenic potential of AT-MSCs was evaluated at
A

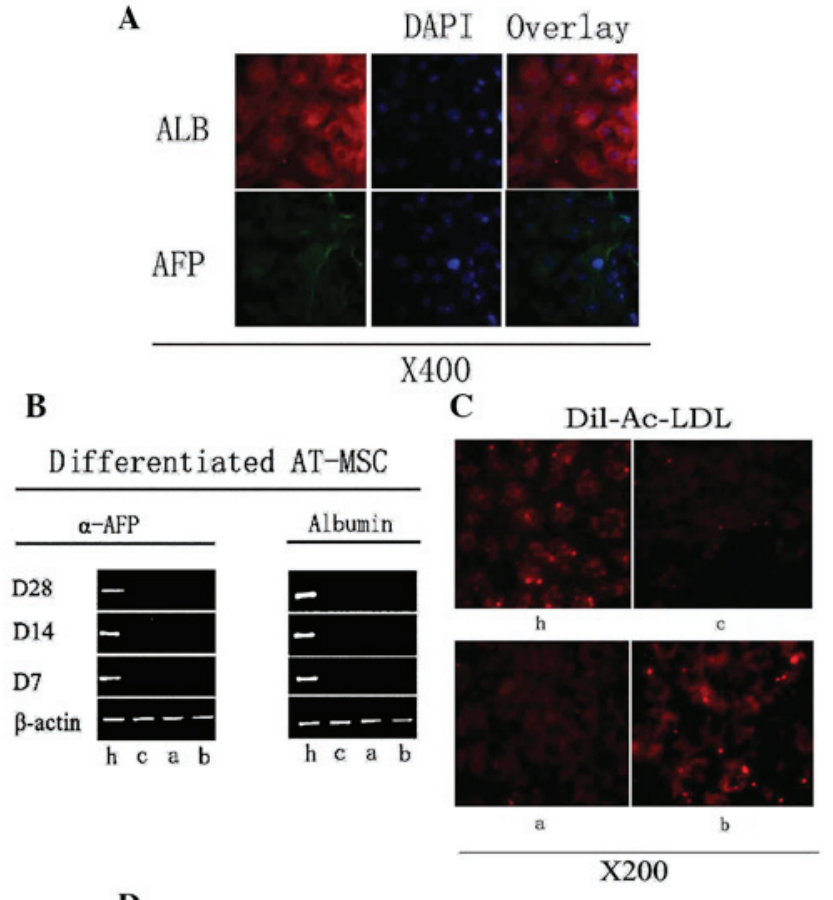

D

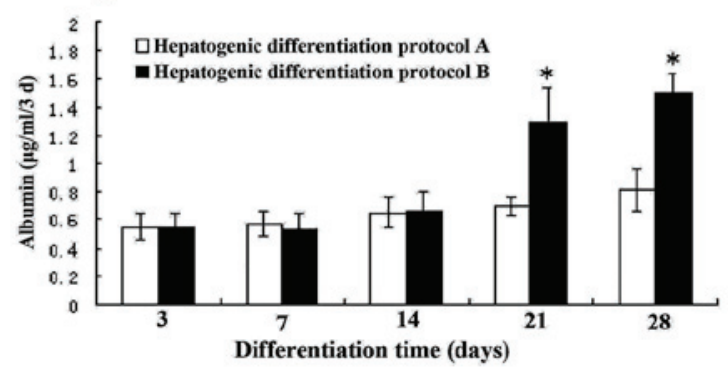

Figure 6. Differentiation potential of AT-MSCs exposed to hepatogenic differentiation medium. (A) ALB expression at day 14 and AFP expression at day 7. (B) Reverse transcription-polymerase chain reaction showed expression levels of ALB and AFP (using $\beta$-actin as a control). (C) AT-MSCs-derived hepatocyte-like cells were analyzed for LDL-uptake at day 28. (D) ALB levels secreted by AT-MSCs-derived hepatocyte-like cells. Data are presented as mean \pm standard deviation and analyzed by Student's t-test $(n=3)$, ${ }^{*} \mathrm{P}<0.05$ protocol A vs. protocol B. h, HepG2; c, control; a, hepatogenic differentiation protocol A; b, hepatogenic differentiation protocol B; AT-MSC, adipose tissue mesenchymal stem cells; ALB, albumin; AFP, $\alpha$-fetoprotein; LDL, low density lipoprotein; Dil-Ac-LDL, dioctadecyl-tetramethyl-indocarbocyanine perchlorate acetylated LDL.

P3-P5. The adipogenic potential was assessed by induction of post-confluent AT-MSCs. Vacuoles appeared after three days of induction, and a consistent cell vacuolation was evident in the cytoplasm. Vacuoles stained strongly for fatty acids with Oil O Red. The lipid vacuoles were identified as bright red inclusions within cells, while the nuclei were stained dark blue with DAPI. Evidence for osteogenic differentiation was observed as morphological changes, which appeared during the first week of subculture. At the end of the 21-day induction period, calcium crystals were clearly visible in culture, and cell differentiation was confirmed by von Kossa staining for calcium. Extracellular calcium mineralization following osteogenic differentiation was visualized as black stains, and the nuclei stained with Neutral Red appeared as pink (Fig. 4).

Hepatic differentiation of AT-MSCs in vivo. 

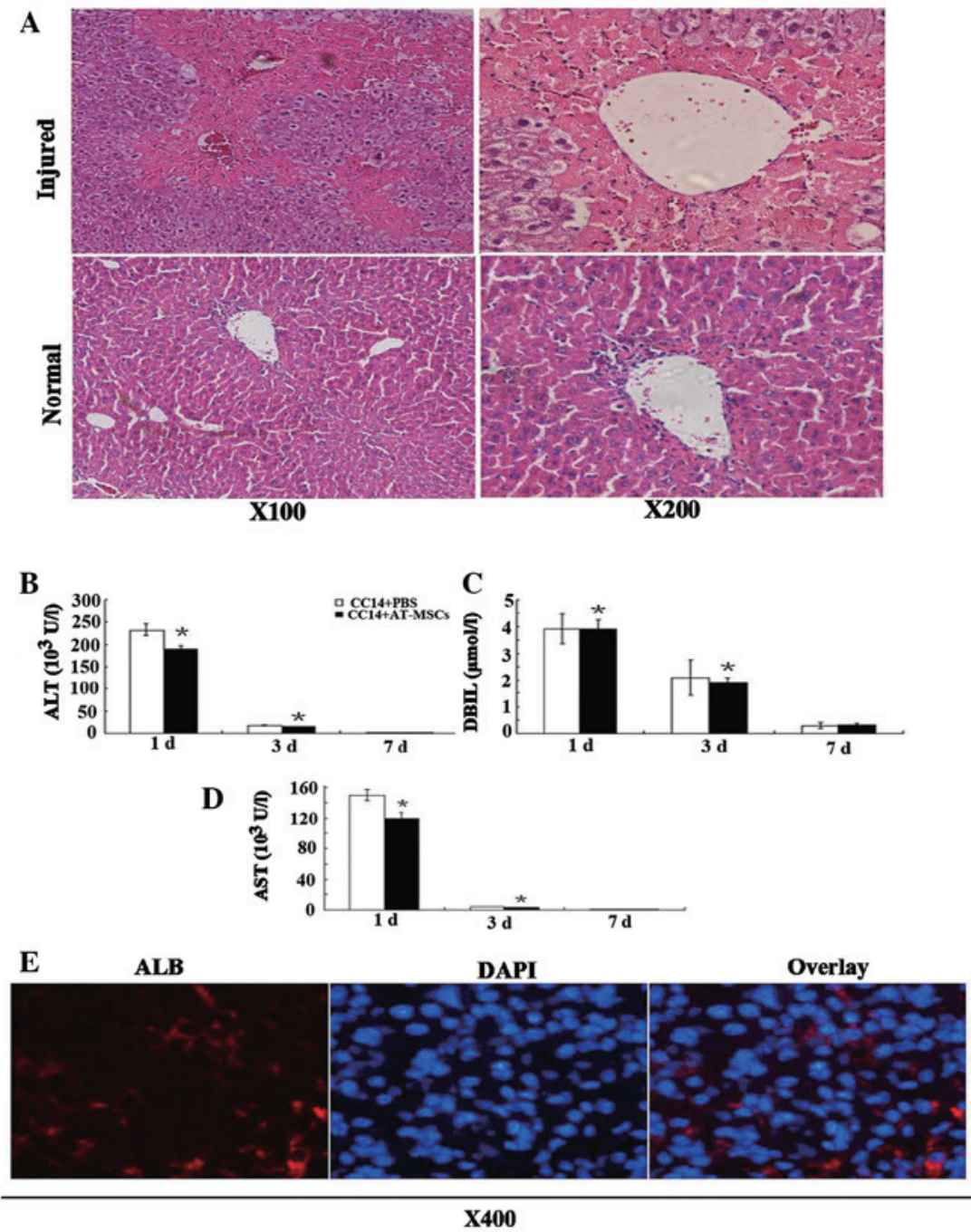

Figure 7. Therapeutic effect of AT-MSC administration. (A) One day following a single injection of $10 \% \mathrm{CCl}_{4}(100 \mu 1 / 20 \mathrm{~g}$ body weight) into the tail vein of nude mice, $\mathrm{H} \& \mathrm{E}$ staining showed pathological changes in liver cells, i.e., heavily damaged central veins of the liver and the morphological characteristics for hepatocytes vanished; (B-D) ALT, AST and DBIL levels decreased dramatically following AT-MSCs administration. Data are presented as mean \pm standard deviation; analyzed by Student's t-test, $\mathrm{n}=3,{ }^{*} \mathrm{P}<0.05$ control vs. AT-MSC-treated group; (E) Human ALB detection in the livers of nude mice one month following injection of $5 \times 10^{5} \mathrm{AT}$-MSCs into $\mathrm{CCl}_{4}$-injured mice. AT-MSC, adipose tissue mesenchymal stem cells; H\&E, hematoxylin and eosin; ALT, alanine aminotransferase; AST, aspartate aminotransferase; DBIL, direct bilirubin; ALB, albumin.

Morphological changes in cultured AT-MSCs. Protocols shown in Fig. 1A and B were used to detect whether FBS could influence the hepatic induction procedure, in particular the morphological changes of the cells. Differentiation medium with or without 2\% FBS was added to confluent AT-MSCs. During the initiation step of hepatic differentiation, the cells treated with serum-free media gradually lost their fibroblastic morphology and developed a broader, flatter shape; these cells subsequently developed a polygonal shape during differentiation (Fig. 5A). The contraction of the cytoplasm progressed further during maturation, and during differentiation the majority of treated cells became dense and round with clear or double nuclei. By contrast, hepatic differentiation medium with $2 \%$ FBS showed no significant morphological changes (Fig. 5A). The induced cells became more dense and elongated, both in the presence and absence of TSA. AT-MSCs were analyzed for glycogen-storage ability using PAS staining. As shown in (Fig. 5B), the serum-free medium-induced hepatocyte-like cells were strongly positive for PAS staining. However, cells with $2 \%$ FBS in the differentiation medium showed relatively weaker staining, and undifferentiated AT-MSCs were weakly positive for PAS staining. It was concluded that the serum-free induction medium was important for the differentiation of AT-MSCs into hepatocyte-like cells, as this dramatically changed the morphology of AT-MSCs from fibroblastic to epithelial.

Function of hepatocyte-like cells derived from AT-MSCs. Assays were performed to investigate the functional competence of AT-MSCs-derived hepatocyte-like cells without serum. At day 14, hepatocyte-like cells expressed both ALB and AFP, as detected by immunostaining using anti-human specific antibodies (Fig. 6A). ALB is a hepatocyte-specific marker of mature functional hepatocytes, while AFP is a marker for immature hepatocytes. Hepatocyte-like cells also showed an ability to store glycogen (Fig. 5B). Furthermore, following 2 weeks of induction, LDL uptake was observed in the hepatocyte-like cells, but did not occur in untreated cells (Fig. 6C). LDL is a lipoprotein that carries cholesterol 
A
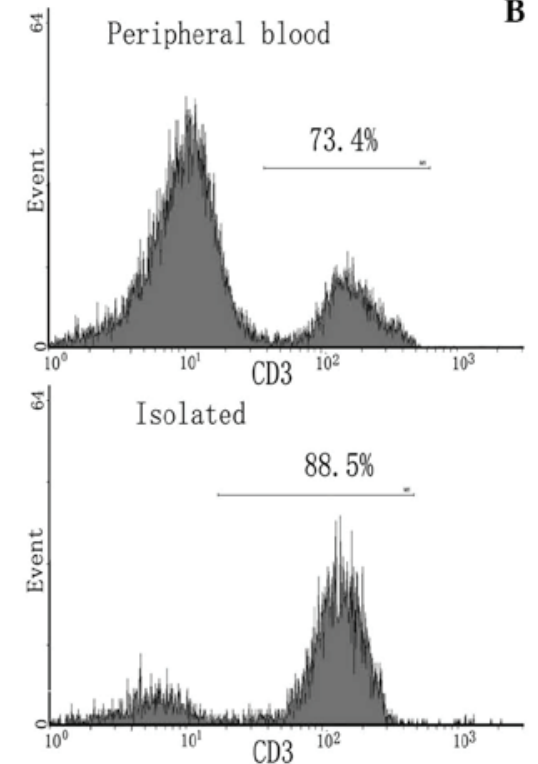

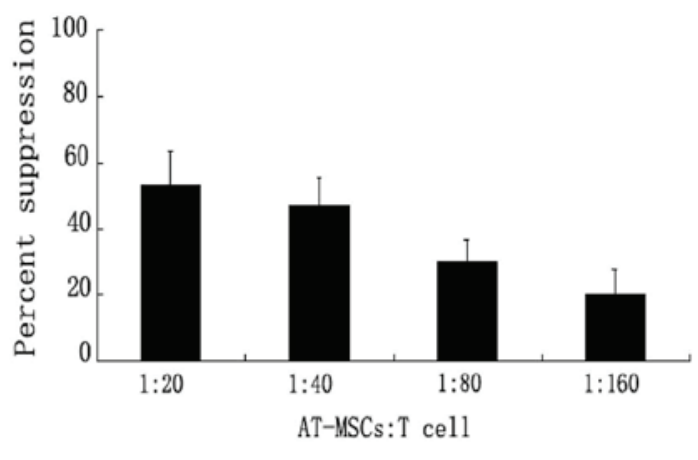

Immune suppressive capacity of AT-MSCs. (A) Ratio of T cells to total lymphocytes increased from $73.4 \%$ in peripheral blood to $88.5 \%$ following isolation. (B) Human AT-MSCs suppressed two-way MLR reactions; the immunosuppressive capacity was dose-dependent. AT-MSC, adipose tissue mesenchymal stem cells; MLR, mixed lymphocyte reaction.

in hepatocytes. During maturation (28 days), the majority of induced hepatocyte-like cells became competent for LDL uptake, a phenotype that was enhanced by addition of TSA to the induction medium (Fig. 6C).

TSA can boost the functions of hepatocyte-like cells derived from AT-MSCs. In an attempt to enhance the in vitro differentiation of AT-MSCs, TSA, a selective and reversible histone deacetylase inhibitor (16), was added to the culture media following exposure of cells to hepatogenic factors over 14 days of incubation. Treatment of undifferentiated AT-MSCs with increased concentrations of TSA resulted in wide-spread cell death and cell detachment (16); therefore, $1.5 \mu \mathrm{M}$ TSA was used in experiments for the present study. Total RNA was isolated at 7,14 and 28 days post-differentiation of AT-MSCs into hepatic lineage, and the expression of several hepatic genes was examined by RT-PCR. Undifferentiated cells were used as negative controls and HepG2 was used as the positive control. The expression pattern of differentiated AT-MSCs from protocol B (Fig. 1B) was used to compare the levels of the ALB and AFP genes relative to human $\beta$-actin at different time-points by RT-PCR. ALB expression was significantly enhanced by induction of differentiation. As AT-MSCs differentiated into hepatocyte-like cells, matured and became more functional, AFP expression gradually diminished, more notably when TSA was added from day 14 onwards (Fig. 6C).

In order to further assess the effect of TSA on differentiated cellular function, the capacity of LDL uptake of hepatocyte-like cells from protocol B was evaluated (Fig. 1B). TSA enhanced the LDL uptake capacity dramatically. When different cell types were co-cultured with LDL for $8 \mathrm{~h}$, the signal indicating LDL uptake in the hepatocyte-like cells in the TSA induction medium was considerably brighter in comparison with cells in the basic induction medium. The negative control cells (undifferentiated AT-MSCs) were considerably darker than the induced cells treated according to protocol A or B (Fig. 6C).

TSA, when added exclusively to AT-MSCs at $100 \%$ confluence and without pre-treatment with hepatogenic medium, was not effective in stimulating mesenchymal-to-hepatic transition. However, AT-MSCs treated with TSA from day 14 onwards exhibited significantly upregulated ALB secretion rates $(\mathrm{P}<0.05$, Student's t-test) when compared with cells in basic differentiation cultures (Fig. 6D).

Transplantation of AT-MSCs into $\mathrm{CCl}_{4}$-injured nude mice results in the improvement of liver function. Transaminase activity and direct bilirubin levels were measured at selected time-points to examine liver function following a single injection of $\mathrm{CCl}_{4}$. Transaminase activity peaked one day post- $\mathrm{CCl}_{4}$ injection, and pathological examination of $\mathrm{H} \& \mathrm{E}$-stained sections revealed large areas of inflammation and hepatocyte denaturation (Fig. 7A). In comparison with normal liver tissue, both transaminase activity and direct bilirubin levels returned to normal seven days following the single injection of $\mathrm{CCl}_{4}$. Transaminase activity corresponded with $\mathrm{H} \& \mathrm{E}$ staining of liver pathology, while the serum ALT, DBIL and AST levels in the $\mathrm{CCl}_{4} / \mathrm{PBS}$ groups were significantly higher than those in the $\mathrm{CCl}_{4} / \mathrm{AT}-\mathrm{MSCs}$ groups (Fig. 7B-D) $(\mathrm{P}<0.05$, Student's t-test). The therapeutic effects of AT-MSCs transplanted into mice with $\mathrm{CCl}_{4}$-induced acute liver injury were demonstrated by the decrease in serum ALT, AST activity and DBIL levels. Although differences in pathologies between $\mathrm{CCl}_{4} / \mathrm{PBS}$ and $\mathrm{CCl}_{4} / \mathrm{AT}$-MSCs groups were not found, the results of the present study showed that transplantation of AT-MSCs had a beneficial effect on liver function in vivo.

AT-MSCs reside in $\mathrm{CCl}_{4}$ injured livers and express liver-specific markers. Liver sections were examined by histochemical immunofluorescence with human ALB-specific 
antibodies (Fig. 7E), which demonstrated the incorporation of AT-MSCs into injured livers. Human ALB-positive cells were found in liver sections following injection of undifferentiated AT-MSCs; however, these cells did not exhibit the typical hepatocyte morphology (Fig. 7E). This indicated that AT-MSCs may have potential clinical application as treatment for acute liver injury.

Low immunogenicity of the AT-MSCS. The potential clinical use of AT-MSCs is attractive due to their low immunogenicity. Both activated and inactivated AT-MSCs lacked expression of co-stimulatory molecules, including CD40, CD54, CD80, CD86, or HLA-DR and HLA-ABC (data not shown). The inability of AT-MSCs to stimulate a T-cell response may be due to an inherently low immunogenicity status, or active immunosuppressive mechanisms mediated by the AT-MSCs. MLR assays with allogeneic $\mathrm{T}$ cells were performed to determine whether the fat-derived cells were immunosuppressive. The ratio of $\mathrm{T}$ cells to total lymphocytes increased from $73.4 \%$ in peripheral blood to $88.5 \%$ following isolation (Fig. 8A). AT-MSCs suppressed MLR cultures in a dose-dependent manner (Fig. 8B); T-cell aggregation was decreased compared with that of with that of controls (data not shown). In addition, AT-MSCs significantly suppressed T-cell proliferation in MLR. These data indicated that allogeneic AT-MSCs may be a potential source of cells for tissue repair or replacement. The present study also has important implications with respect to the ready availability of adult stem cells for clinical applications, and to the practical and commercial aspects of their manufacture and quality assurance. AT-MSCs are not only inherently non-immunogenic and have the ability to suppress proliferation of alloantigen- or mitogen-stimulated $\mathrm{T}$ cells, but they can also suppress immunoglobulin production by mitogen-stimulated B cells (27). The low immunogenicity and immunosuppressive characteristics show that human adipose tissue may be an ideal source for cell transplantation (27-29).

\section{Discussion}

AT-MSCs have great potential for clinical applications in regenerative medicine. Transplantation of AT-MSCs may provide an easier, more efficient and safer method for the treatment of patients with liver disease than whole organ transplantation (17). Adipose tissue can be obtained in large quantities with minimally invasive procedures and AT-MSCs can be easily isolated and cultured in vitro (17-19). AT-MSCs have a broader differentiation potential than previously anticipated and can differentiate into all mesodermal lineage cells, including osteocytes, adipocytes and chondrocytes $(20,21)$. AT-MSCs have characteristics similar to those of BMSCs; AT-MSCs have the ability to differentiate into osteogenic and adipogenic cells in vitro; however they do not express hematopoietic cell antigens. The mechanism of MSC differentiation into hepatocyte-like cells in vitro has not been determined extensively: Baertschiger et al (22) reported that co-culturing with Huh-7 cells was sufficient to induce hepatic differentiation. Chien et al (23) found that coating culture plates with different proteins affected human placenta-derived multi-potent cells, which when cultured in differentiation medium on poly-L-lysine-coated plates, changed morphology and became polygonal in shape. These morphological changes were less obvious when cells were cultured on fibronectin-coated plates.

TSA is a trigger for the further differentiation of human MSCs towards endodermal lineages (24). High levels of TSA can induce cell apoptosis and promote differentiation by inducing cell cycle arrest during the G0/G1 and G1/S phases (25). Vinken et al (25) reported that TSA counteracted the loss of liver-specific functions in primary rat hepatocytes (26) and enhanced intercellular communication through gap junctions. In the present study, TSA significantly boosted the function of hepatocyte-like cells derived from AT-MSCs, regardless of increases in the ALB mRNA and protein levels, in addition to enhancing their glycogen storage abilities.

In vivo, the transplanted cells were exposed to a dynamic host microenvironment laden with soluble mediators and immunoreactive cells. The present study hypothesized that at least three ubiquitous microenvironmental factors may affect the differentiation and function of the MSCs. Interleukin-1 $\alpha(\mathrm{IL}-1 \alpha)$ is a key regulator of hematopoiesis and the inflammatory process (30). Recent studies on mice have shown that MSCs have an inherent ability to counteract the deleterious inflammatory effects of IL-1 $\alpha$ in injured tissues (31) In addition, tumor necrosis factor $\alpha$, which has been shown to increase chemotaxis of MSCs, would also be clinically important if the efficiency of concentrating MSCs to the site of tissue injury was improved (32). Furthermore, stromal cell-derived factor-1 $\alpha$ is a promoter of non-specific MSC migration $(33,34)$. Therefore, co-therapy with a pharmacological agent, such as a cytokine receptor antagonist, may negate the deleterious effects of the microenvironment and optimize the therapeutic potential of MSCs $(31,35)$. Human BMSCs used for transplantation have been expanded without significant loss of their differentiation capacities. Following transplantation into unconditioned adult mice, BMSCs not only migrated to the bone marrow but also into other tissues. Impairment of the tissue was shown to cause increased BMSC implantation not only in bone marrow and muscle but it was also shown to lead to further engraftment in the brain, heart and liver (36).

Oyagi et al (37) reported that BMSCs reduced hepatocyte apoptosis and promoted cell proliferation. In addition, they showed that autologous MSC transplantation prolonged the survival of dogs (38) and swine (39) receiving living donor liver transplantation. The present study clearly demonstrated that AT-MSCs can ease mouse acute hepatic injury in vivo by decreasing inflammation and apoptosis as well as increasing proliferation and recovery (as evidenced by decreased ALT, AST and DBIL levels). It was demonstrated that AT-MSCs are a novel source of cells that may be used to treat hepatic injury and/or dysfunction. The mechanisms by which hepatic differentiation occurs in vivo and hepatic function is restored, however, are still not fully elucidated. Oyagi et al (37) reported that BMSCs secreted HGF and suppressed inflammation when transplanted into $\mathrm{CCl}_{4}$-injured rats. $\mathrm{HGF}$ had the capacity to induce hepatic differentiation and suppress hepatocyte death (40). Silva et al (41) reported that the transplantation of MSCs reduced fibrosis through the secretion of cytokines, in particular vascular endothelial growth factor. BMSCs have the capacity to differentiate into hepatocyte-like cells in response to growth factor stimulation (37). Results of 
the present study showed that AT-MSCs can only reside in $\mathrm{CCl}_{4}$-injured livers, suggesting that hepatic differentiation of AT-MSCs may be induced by HGF and/or other cytokines secreted by the $\mathrm{CCl}_{4}$-injured livers. It remains elusive whether MSCs contribute to tissue repair by differentiation into tissue-specific cell types, or whether they produce trophic factors at the site of injury, which can stimulate tissue repair $(42,43)$. MSCs are responsive to their environment, adapting function to local circumstances, and immunosuppressive properties appear to be induced under inflammatory conditions (43). Modification of the culture medium could modulate the properties of MSCs, for example, by enhancing immunosuppressive function and reducing susceptibility for lysis by cytotoxic T cells (44).

In conclusion, ALB was detected in the livers of the $\mathrm{CCl}_{4}$-injured mice one month post-transplantation. This suggested that transplantation of the human AT-MSCs could relieve the impairment of acute $\mathrm{CCl}_{4}$-injured livers in nude mice. This therefore implied that adipose tissue was a source of multipotent stem cells which had the potential to differentiate into mature, transplantable hepatocyte-like cells in vivo and in vitro. In addition, the present study determined that TSA was essential to promoting differentiation of human MSC towards functional hepatocyte-like cells. The relief of liver injury following treatment with AT-MSCs suggested their potential as a novel therapeutic method for liver disorders or injury. Human AT-MSCs may become a useful source for hepatocyte regeneration and may provide an alternative to liver transplantation.

\section{Acknowledgements}

This study was funded by the National Natural Science Foundation of China (no.30872484) and the Science Foundation of Nanjing Medical University (no. 2012NJMU134).

\section{References}

1. van Poll D, Parekkadan B, Cho CH, et al: Mesenchymal stem cell-derived molecules directly modulate hepatocellular death and regeneration in vitro and in vivo. Hepatology 47: 1634-1643, 2008.

2. Kashofer $\mathrm{K}$ and Bonnet $\mathrm{D}$ : Gene therapy progress and prospects: stem cell plasticity. Gene Ther 12: 1229-1234, 2005.

3. Kern S, Eichler H, Stoeve J, Klüter H and Bieback K: Comparative analysis of mesenchymal stem cells from bone marrow, umbilical cord blood, or adipose tissue. Stem Cells 24: 1294-1301, 2006.

4. Devine SM, Cobbs C, Jennings M, Bartholomew A and Hoffman R: Mesenchymal stem cells distribute to a wide range of tissues following systemic infusion into nonhuman primates. Blood 101: 2999-3001, 2003.

5. Tepliashin AS , Chupikova NI, Korzhikova SV, et al: Comparative analysis of cell populations with a phenotype similar to that of mesenchymal stem cells derived from subcutaneous fat. Tsitologiia 47: 637-643, 2005 (in Russian).

6. Malhi H , Irani AN, Gagandeep S and Gupta S: Isolation of human progenitor liver epithelial cells with extensive replication capacity and differentiation into mature hepatocytes. J Cell Sci 115: 2679-2688, 2002.

7. Young HE, Steele TA, Bray RA, et al: Human reserve pluripotent mesenchymal stem cells are present in the connective tissues of skeletal muscle and dermis derived from fetal, adult, and geriatric donors. Anat Rec 264: 51-62, 2001.

8. Lowes KN, Croager EJ, Olynyk JK, Abraham LJ and Yeoh GC: Oval cell-mediated liver regeneration: Role of cytokines and growth factors. J Gastroenterol Hepatol 18: 4-12, 2003.
9. Zuk PA, Zhu M, Ashjian P, et al: Human adipose tissue is a source of multipotent stem cells. Mol Biol Cell 13: 4279-4295, 2002.

10. Lagasse E, Connors H, Al-Dhalimy M, et al: Purified hematopoietic stem cells can differentiate into hepatocytes in vivo. Nat Med 6: 1229-1234, 2000.

11. Alison MR, Poulsom R, Jeffery R, et al: Hepatocytes from non-hepatic adult stem cells. Nature 406: 257, 2000.

12. Herzog EL, Chai L and Krause DS: Plasticity of marrow-derived stem cells. Blood 102: 3483-3493, 2003.

13. Vassilopoulos G, Wang PR and Russell DW: Transplanted bone marrow regenerates liver by cell fusion. Nature 422: 901-904, 2003.

14. Wang X, Willenbring H, Akkari Y, et al: Cell fusion is the principal source of bone-marrow-derived hepatocytes. Nature 422: 897-901, 2003.

15. Wagner W, Wein F, Seckinger A, et al: Comparative characteristics of mesenchymal stem cells from human bone marrow, adipose tissue, and umbilical cord blood. Exp Hematol 33: 1402-1416, 2005.

16. Snykers S, Vanhaecke T, De Becker A, et al: Chromatin remodeling agent trichostatin A: a key-factor in the hepatic differentiation of human mesenchymal stem cells derived of adult bone marrow. BMC Dev Biol 7: 24, 2007.

17. Yamamoto H, Quinn G, Asari A, et al: Differentiation of embryonic stem cells into hepatocytes: biological functions and therapeutic application. Hepatology 37: 983-993, 2003.

18. Kim JW, Kim SY, Park SY, et al: Mesenchymal progenitor cells in the human umbilical cord. Ann Hematol 83: 733-738, 2004.

19. Reyes M, Lund T, Lenvik T, et al: Purification and ex vivo expansion of postnatal human marrow mesodermal progenitor cells. Blood 98: 2615-2625, 2001.

20. Teratani T, Yamamoto H, Aoyagi K, et al: Direct hepatic fate specification from mouse embryonic stem cells. Hepatology 41: 836-846, 2005.

21. Yamamoto Y, Teratani T, Yamamoto $\mathrm{H}$, et al: Recapitulation of in vivo gene expression during hepatic differentiation from murine embryonic stem cells. Hepatology 42: 558-567, 2005.

22. Baertschiger RM, Serre-Beinier V, Morel P, et al: Fibrogenic potential of human multipotent mesenchymal stromal cells in injured liver. PLoS One 4: e6657, 2009.

23. Chien $\mathrm{CC}$, Yen BL, Lee FK, et al: In vitro differentiation of human placenta-derived multipotent cells into hepatocyte-like cells. Stem Cells 24: 1759-1768, 2006.

24. Herold C, Ganslmayer M,Ocker M, et al: The histone-deacetylase inhibitor Trichostatin A blocks proliferation and triggers apoptotic programs in hepatoma cells. J Hepatol 36: 233-240, 2002.

25. Vinken M, Henkens T, Vanhaecke T, et al: Trichostatin a enhances gap junctional intercellular communication in primary cultures of adult rat hepatocytes. Toxicol Sci 91: 484-492, 2006.

26. Henkens T, Papeleu P, Elaut G, et al: Trichostatin A, a critical factor in maintaining the functional differentiation of primary cultured rat hepatocytes. Toxicol Appl Pharmacol 218: 64-71, 2007.

27. Bochev I, Elmadjian G, Kyurkchiev D, et al: Mesenchymal stem cells from human bone marrow or adipose tissue differently modulate mitogen-stimulated B-cell immunoglobulin production in vitro. Cell Biol Int 32: 384-393, 2008.

28. McIntosh K, Zvonic S, Garrett S, et al: The immunogenicity of human adipose-derived cells: temporal changes in vitro. Stem Cells 24: 1246-1253, 2006.

29. Battiwalla M and Hematti P: Mesenchymal stem cells in hematopoietic stem cell transplantation. Cytotherapy 11: 503-515, 2009.

30. Dinarello CA: The interleukin-1 family: 10 years of discovery. FASEB J 8: 1314-1325, 1994.

31. Ortiz LA, Dutreil M, Fattman C, et al: Interleukin 1 receptor antagonist mediates the anti-inflammatory and antifibrotic effect of mesenchymal stem cells during lung injury. Proc Natl Acad Sci USA 104: 11002-11007, 2007.

32. Ponte AL, Marais E, Gallay N, et al: The in vitro migration capacity of human bone marrow mesenchymal stem cells: comparison of chemokine and growth factor chemotactic activities. Stem Cells 25: 1737-1745, 2007.

33. Potian JA, Aviv H, Ponzio NM, Harrison JS and Rameshwar P. Veto-like activity of mesenchymal stem cells: functional discrimination between cellular responses to alloantigens and recall antigens. J Immunol 171: 3426-3434, 2003.

34. Tang J, Wang J, Yang J, et al: Mesenchymal stem cells over-expressing SDF-1 promote angiogenesis and improve heart function in experimental myocardial infarction in rats. Eur J Cardiothorac Surg 36: 644-650, 2009. 
35. Greco SJ and Rameshwar P: Microenvironmental considerations in the application of human mesenchymal stem cells in regenerative therapies. Biologics 2: 699-705, 2008.

36. Mouiseddine M, François S, Semont A, et al: Human mesenchymal stem cells home specifically to radiation-injured tissues in a non-obese diabetes/severe combined immunodeficiency mouse model. Br J Radiol 80 Spec No 1: S49-55, 2007.

37. Oyagi S, Hirose M, Kojima M, et al: Therapeutic effect of transplanting HGF-treated bone marrow mesenchymal cells into $\mathrm{CCl}_{4}$-injured rats. J Hepatol 44: 742-748, 2006.

38. Pan MX, Hou WL, Zhang QJ, et al: Infusion of autologous mesenchymal stem cells prolongs the survival of dogs receiving living donor liver transplantation. J South Med Univ (in Chinese).

39. Kuo YR, Goto S, Shih HS, et al: Mesenchymal stem cells prolong composite tissue allotransplant survival in a swine model. Transplantation 87: 1769-1777, 2009.
40. Matsuda Y, Matsumoto K, Ichida T and Nakamura T: Hepatocyte grow th factor suppresses the onset of liver cirrhosis and abrogates lethal hepatic dysfunction in rats. J Biochem 118: 643-649, 1995.

41. Silva GV, Litovsky S, Assad JA, et al: Mesenchymal stem cells differentiate into an endothelial phenotype, enhance vascular density, and improve heart function in a canine chronic ischemia model. Circulation 111: 150-156, 2005.

42. Phinney DG and Prockop DJ: Concise review: mesenchymal stem/multipotent stromal cells: the state of transdifferentiation and modes of tissue repair - current views. Stem Cells 25: 2896-2902, 2007.

43. Krampera M,Cosmi L, Angeli R, et al: Role for interferon-gamma in the immunomodulatory activity of human bone marrow mesenchymal stem cells. Stem Cells 24: 386-398, 2006.

44. Rasmusson I, Uhlin M,Le Blanc K and Levitsky V: Mesenchymal stem cells fail to trigger effector functions of cytotoxic T lymphocytes. J Leukoc Biol 82: 887-893, 2007. 